\title{
Pulsations and declines of RCB stars
}

\author{
P.L. Cottrell ${ }^{1}$, W.A. Lawson ${ }^{2}$ \\ ${ }^{1}$ Mount John University Observatory, Dept of Physics \& Astronomy, \\ University of Canterbury, Christchurch, New Zealand, \\ ${ }^{2}$ Dept of Physics, University College ADFA, UNSW, Canberra, Australia
}

\begin{abstract}
We have continued to observe many of the $\mathrm{R}$ Coronae Borealis (RCB) stars and other related hydrogen-deficient carbon stars. We wish to more fully investigate the photometric and spectroscopic properties of these peculiar stars.

The UBVRI photometric data (acquired at Mount John University Observatory, MJUO) are being used to investigate whether the periods determined by Lawson et al. (1990), based on time intervals of up to $1100 \mathrm{~d}$, are still evident in the larger datasets which now cover time intervals of up to $2200 \mathrm{~d}$.

The long time baseline of photometric observations that we have been able to acquire has enabled us to accumulate an extensive database of decline photometry. These are used to investigate links with the pulsations at maximum light in order to get a better understanding of this remarkable phenomenon.
\end{abstract}

\section{Introduction}

Up until a decade ago, the RCB stars had been regarded as an astronomical enigma and objects that were the subject of only occasional and brief investigation during the unusual dimming events that these stars undergo. However, these investigations have often only been undertaken once the object has been in decline for a number of days or weeks. Although this can provide valuable information about the evolution of the obscuring material, any understanding of the triggering mechanism is lost.

\section{Pulsations \& Declines}

(a) Pulsations at maximum light (U Aqr \& RY Sgr)

U Aqr was observed to have alternating deep and shallow minima in the data obtained in 1986 and 1987. This effect disappeared during the subsequent 2 years where there was little distinctly periodic photometric variation in the $V$ light curve. The amplitude of the pulsations increased again in 1991. The frequency analysis shows that the 1991 observations are in phase with the previous alternations in light output (see figure 1), similar to the RV Tauri stars (see Pollard et al., this conference).

RY Sgr has continued its semi-regular pulsations, despite the decline during 1990. There are still 2 dominant periods (52- d and 37-d) in the 1991 photometric data (see figure 2). Photometry of RY Sgr obtained at MJUO since 1986 indicates that 
the amplitude of the 37-d mode has remained essentially constant ( $0.19 \pm 0.03 \mathrm{mag})$, whereas the amplitude of the $52-\mathrm{d}$ mode varies by a factor of 4 (between 0.06 and $0.24 \mathrm{mag}$ ). The varying amplitude of this mode is almost certainly the reason for the semi-regular appearance of the light curve of RY Sgr.
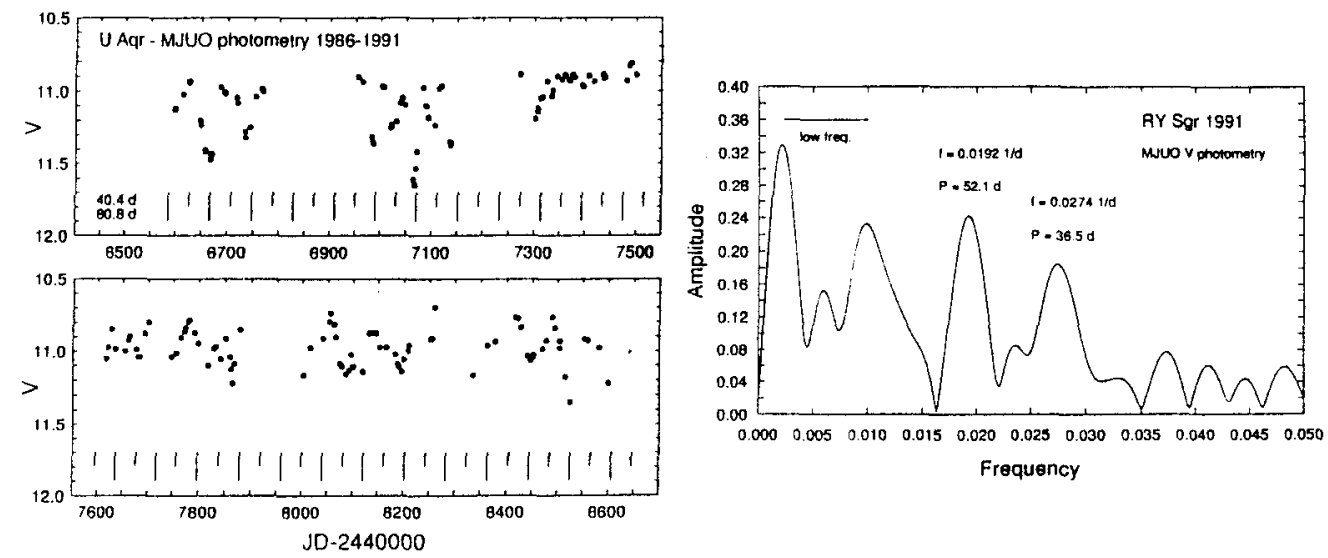

Figure 1 MJUO $V$ photometry of U Aqr obtained since 1986. The 40.4-d and 80.8-d periodicities have been determined from the Fourier analysis of the light curve.

Figure 2 The amplitude spectrum for the 1991 photometry of RY Sgr. The rise from the decline in 1990 shows as low frequency peaks. The other two dominant periodicities, similar to those found in previous years, are indicated.

(b) Pulsation-decline links (RY Sgr \& V8.54 Cen)

Pugach (1977) noted that declines of RY Sgr occurred within a narrow range of phase, near maximum light of the $37-\mathrm{d}$ pulsation mode. A similar relationship was recently found for V854 Cen, which has declines occurring near the maximum of its 43.2-d periodicity (Lawson et al. 1992). These discoveries infer dust formation close to the star (to preserve the phase coherency) and at near-photospheric temperatures. The 'standard' model for mass-loss in RCB stars (see Feast 1990) considers mass-loss via some ejection mechanism with dust formation at $\sim 20 R_{*}$. A pulsation-decline link under these conditions would be highly unlikely.

Acknowledgments: We would like to thank Alan Gilmore and Pam Kilmartin for the acquisition of the photometric data. PLC acknowledges the financial support of an Erskine Fellowship from the University of Canterbury and the hospitality of the Department of Physcis, University College ADFA, where this paper was written.

\section{References:}

Feast, M.W., 1990, Astr. Soc. of Pacific Conf. Series 11, 538.

Lawson, W.A., Cottrell, P.L., Kilmartin, P.M., Gilmore, A.C., 1990, MNRAS 247, 91.

Lawson, W.A., Cottrell, P.L., Gilmore, A.C., Kilmartin, P.M., 1992, MNRAS 256, 347.

Pugach, A.F., 1977, IBVS No. 1277. 